\title{
THE RIEMANN MULTIPLE-SPACE AND ALGEBROID FUNCTIONS*
}

BY

\author{
B. O. KOOPMAN $\dagger$ AND A. B. BROWN
}

1. Introduction. The present paper considers the extension of the Riemann surface to the case of several complex variables. The resulting configuration will be called Riemann multiple-space $\S$ (R. M. S.), and the first object is to give its construction, or definition. It is then shown that the R. M. S. is a generalized manifold. $\|$ The property of being a generalized manifold is shown to be a topologically invariant property of a complex, and a simple characterization of a $G M_{n}$ is given. The locus of non-spherical points $\Phi$ of the R. M.S.

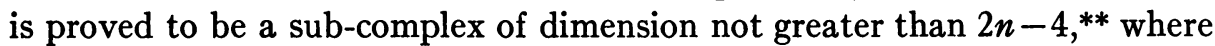
$n$ is the number of independent variables; an example due to Osgood $\dagger \dagger$ shows that it can actually attain that dimensionality.

2. Properties of the generalized manifold. We prove certain properties which are needed in what follows.

Leмma 1. A generalized n-manifold is a simple n-circuit. $¥ \ddagger$

* Presented to the Society, October 28, 1933; received by the editors November 28, 1933.

† Some of the results of the present paper were announced in preliminary form in the abstract bearing the same title presented by B. O. Koopman (at that time National Research Council Fellow) in the Bulletin of the American Mathematical Society, vol. 33 (1927), p. 406.

‡ For a treatment of the case of one independent variable, see $\mathrm{H}$. Weyl, Die Idee der Riemannschen Fläche, 2d edition, Leipzig, 1923.

$\$$ Terms often used are Riemann hypersurface or Riemann space; but it seems undesirable to use these, inasmuch as their use in the present connection involves a contradiction with other standard mathematical usage.

\| O. Veblen, A nalysis Situs, chapter III, pp. 95-96 in second edition; Colloquium Series, vol. 5, part 2, New York, 1931. A generalized manifold of $n$ dimensions $\left(G M_{n}\right)$ is defined as the set of points on an $n$-circuit such that the cells of higher dimensions incident with any given $i$-cell have the incidence relations of a $G M_{n-i-1}$. The only $G M_{0}$ is a pair of 0 -cells.

Terminology will be as defined in Veblen, or as in Lefschetz's Topology, Colloquium Series, vol. 12, New York, 1930. (Lefschetz I.)

An $n$-circuit is an $n$-complex which (1) is the closure of its $n$-cells; (2) has an even number of $n$-cells incident with each of its $(n-1)$-cells; (3) contains no proper sub-complex satisfying (1) and (2).

If A point of a $k$-complex will be called a spherical point if it has a neighborhood on the complex which is homeomorphic to a $k$-cell.

** Note that this result does not of itself imply that the R.M.S. is a generalized manifold, nor does the latter imply the former.

†† W. F. Osgood, Lehrbuch der Funktionentheorie, vol. 2, first part, chapter 2, \$21. (Osgood II.)

$\ddagger \ddagger$ A simple $n$-circuit is an $n$-circuit each of whose $(n-1)$-cells is incident with exactly two of its n-cells. 
This lemma is stated for convenience in reference. It follows from the facts that the $n$-manifold is an $n$-circuit and that a $G M_{0}$ is a pair of 0 -cells.

Lemma 2. A definition of $G M_{n}, n>0$, equivalent to the original one, is the following. $A G_{n}$ is a connected n-complex $K_{n}$ such that the cells of higher dimensions incident with any given $i$-cell have the incidence relations of a $G M_{n-i-1}$.

Using induction, we assume the lemma proved for dimensions less than $n$. As our proof will require no assumption for the case $n=1$, it remains only to prove the lemma for general $n$ under the assumption of the induction.

The new definition differs from the original one only in the replacement of " $n$-circuit" by "connected $n$-complex." As any $n$-circuit is connected, we need merely show that under the new definition a $G M_{n}$ is an $n$-circuit. As the cells of higher dimensions incident with any $i$-cell have the incidence relations of an $(n-i-1)$-complex, it follows that every point of $K_{n}$ is on the closure of at least one $n$-cell. As the incidence relations between the cells incident with any $(n-1)$-cell $E_{n-1}$ are those of a $G M_{0}$, it follows that $E_{n-1}$ is incident with just two $n$-cells. Hence $K_{n}$ is an $n$-cycle each of whose $(n-1)$ cèlls is incident with just two $n$-cells.

If $K_{n}$ were not an $n$-circuit there would be two sub-complexes $M_{n}^{1}$ and $M_{n}^{2}$, each an $n$-cycle, containing all the $n$-cells of $K_{n}$ but having no common $n$-cells. As $M_{n}$ is connected, $M_{n}^{1}$ and $M_{n}^{2}$ would have at least one common cell, say an $i$-cell $E_{i}$. As the lemma is assumed true for dimensions less than $n$, the cells $E^{i}$ of higher dimensions, of $K_{n}$, incident with $E_{i}$, would have the incidence relations of an $(n-i-1)$-circuit $c_{n-i-1}$. Since $M_{n}^{1}$ and $M_{n}^{2}$ are $n$ cycles, those of the cells of the set $E^{i}$ belonging to $M_{n}{ }^{i}$ would have the incidence relations of an $(n-i-1)$-cycle $c_{n}^{i}{ }_{i-1}, j=1,2$, which could be considered as a sub-complex of $c_{n-i-1}$. But that is impossible, as an $(n-i-1)$-circuit cannot have two sub-complexes each of which is an $(n-i-1)$-cycle, and distinct. Hence $K_{n}$ must be an $n$-circuit, and the proof is complete.

Lemma 3. A complex $K_{n}$ is a generalized manifold if and only if it is connected, is the closure of its $n$-cells, and satisfies the following condition: If $K_{n}^{s}$ is the set of spherical points of $K_{n}, K_{n}$ is locally connected* by curves which can be taken in $K_{n}^{s}$ whenever their end points are in $K_{n}^{s}$.

To prove the necessity, let $P$ be any point of $K_{n}$, and $E_{i}$ the cell of $K_{n}$ on which it lies. Let $N$ be any neighborhood of $P$ on $K_{n}$, and $N^{\prime} \subset N$ the neighborhood consisting of all the cells of $K_{n}{ }^{\prime}$ on whose closures $P$ lies, where $K_{n}^{\prime}$ is the complex obtained by subdividing $K_{n}$ regularly enough times so

\footnotetext{
* Local connectedness in the ordinary sense is meant; in the terminology of Lefschetz I this means local 0 -connectedness.
} 
that such an $N^{\prime}$ exists. Let $P_{1}$ and $P_{2}$ be any two points in $N^{\prime}$. Now we consider $E_{i}$ and all the cells of higher dimensions that are incident with it. The latter have the incidence relations of a generalized $(n-i-1)$-manifold. As this manifold is, according to Lemma 1 , a simple circuit, we can name a sequence of $(n-i-1)$ - and $(n-i-2)$-cells such that the corresponding sequence of $n$ - and $(n-1)$-cells of $K_{n}$ has the following properties: (1) each cell is incident with the adjacent ones in the sequence; (2) $P_{1}$ is on the first cell or on its boundary; (3) $P_{2}$ is on the last cell or on its boundary. The rest of the proof is obvious.

To prove the sufficiency, suppose the condition satisfied and let $i$ be an integer such that for every $E_{r}, r<i$, the incident cells $E^{r}$ of higher dimensions have the incidence relations of a $G M_{n-r-1}$. We shall prove that the property holds also when $r=i$. Let $E_{i}$ be any $i$-cell. The cells $E^{i}$ of higher dimensions incident with $E_{i}$ have the incidence relations of a complex $k_{n-i-1}$, since $K_{n}$ is the closure of its $n$-cells. Let $E_{i+j}$ be any $(i+j)$-cell incident with $E_{i}$. By hypothesis, the cells $E^{i+j}$ incident with $E_{i+j}$ have the incidence relations of a $G M_{n-i-i-1}$. Now in considering $k_{n-i-1}, E_{i+j}$ corresponds to a. $(j-1)$-cell $e_{j-1}^{i+j}$ of $k_{n-i-1}$. As the incidence relations of the cells of $E^{i+j}$ are the same as the incidence relations of the corresponding cells of $k_{n-i-1}$ incident with $e_{j-1}^{i+j}$, it follows that the latter relations are likewise those of a $G M_{n-i-j-1}$. Thus $e_{j-1}^{i+j}$ satisfies the condition imposed on a $(j-1)$-cell and its incident cells of higher dimensions on $k_{n-i-1}$ in order that $k_{n-i-1}$ should be a $G M_{n-i-1}$. Since a similar statement can be made for any $(i+j)$-cell incident with $E_{i}, j>0$, it follows from Lemma 2 that each connected part of $k_{n-i-1}$ is a $G M_{n-i-1}$ *

Now if $k_{n-i-1}$ were not connected, we could let $P^{1}$ and $P^{2}$ be points on $n$-cells of $K_{n}$ corresponding to $(n-i-1)$-cells of two unconnected parts of $k_{n-i-1}$, sufficiently near to some point $P$ of $E_{i}$ to satisfy the condition of Lemma 3 for some neighborhood $N$ of $P$ containing no points on the boundary of $E_{i}$. Then a curve $C$ would exist joining $P^{1}$ to $P^{2}$ on $K_{n}{ }^{8}$ and in $N$. Then $C$ would contain a point $Q$ on $E_{i}$, as no cell of the group corresponding to the first part of $k_{n-i-1}$ could be incident with any cell of the group corresponding to the second part. Since $Q$ would have an $n$-cell neighborhood, by the invariance of the combinatorial manifold $\dagger$ it follows that $k_{n-i-1}$ would be a combinatorial $(n-i-1)$-sphere. As the latter is connected, we would then have a contradiction to the hypothesis that $k_{n-i-1}$ is not connected. Consequently it is connected, and therefore a $G M_{n-i-1}$.

* Cf. Veblen, loc. cit., pp. 96-97.

† E. R. van Kampen. For references, see Lefschetz I. The linked complex of $E_{i}$ has the construction of a regular subdivision of $k_{n-i-1}$. We have not used linked complexes in the proofs, as they would have necessitated longer proofs. 
It now follows by induction that for every $r$-cell $E_{r}, r=0,1, \cdots, n-1$, the incident cells of higher dimensions have the incidence relations of a $G M_{n-r-1}$. Since $K_{n}$ is connected, it follows from Lemma 2 that $K_{n}$ is a $G M_{n}$, and the proof of Lemma 3 is complete.

The following combinatorial characterization of the $G M_{n}$ is an easily proved consequence of Lemma 3.

Corollary. A necessary and sufficient condition that a complex $K$ be a $G M_{n}$ is that it have the following properties: firstly, it is connected; secondly, every cell is an $n$-cell or on the boundary of an $n$-cell; thirdly, given any $i$-cell $E_{i}$, $i<n$, and any two $n$-cells $E_{n}{ }^{1}$ and $E_{n}{ }^{2}$ incident with $E_{i}$, there exists a sequence of cells of $K$ having the following properties: (1) $E_{n}^{1}$ is the first cell of the sequence and $E_{n}^{2}$ is the last; (2) the cells of the sequence are alternately $n$-cells and $(n-1)$ cells; (3) each cell of the sequence is incident with the adjacent cells of the sequence; (4) all the cells of the sequence are incident with $E_{i}$.

This result might be described more briefly in the following terms. $A$ generalized n-manifold is a connected n-complex which is locally a simple $n$ circuit.*

3. The Riemann multiple-space. Let a region $R$ be given in the $(2 n+2)$ space of the complex variables $w, z_{1}, \cdots, z_{n}$, together with a function $F\left(w, z_{1}, \cdots, z_{n}\right)=F(w, z)$ with the following properties: (1) $F$ is single-valued and analytic at all points of $R$; (2) $F=0$ for some points in $R$; (3) if we continue analytically from a point $P$ at which $F=0$, over a path which may go outside $R$, and return to the point $P$, then if the continued function vanishes at $P$ it must be identical with the original function $F(w, z)$ at $P$; (4) $F$ is irreducible, that is, it is not a product of two functions each satisfying the preceding conditions and having the same locus of points when equated to zero. $\dagger$

Given a point $P$ on the locus $F=0$, let $F$ be factored into a product of irreducible analytic factors $F^{i}$, each vanishing at $P$. It will be proved below that no two of the $F^{i}$ can be equivalent at $P$. $\ddagger$

The Riemann multiple-space for the locus $F=0$ in $R$ is defined as the following Hausdorff space. A point $P$ on $F=0$ together with one of the irreducible functions $F^{i}$ at $P,\left(P, F^{i}\right)$, constitute a point of the space. If $F^{i}$ and $F^{i}$ are equivalent at $P,\left(P, F^{i}\right)$ and $\left(P, F^{i}\right)$ are the same point of the $R . M . S . A$ neighborhood of $\left(P, F^{i}\right)$ consists of the set of points $\left(Q, F^{j}\right)$ for which $Q$ is in a neighborhood of $P$ on $F=0$ in $(w, z)$-space, and $F^{i}=F^{i} \Phi$ at and near $Q$, where $\Phi$ is analytic at $Q$.

* Because of this fact, it may seem that we could have dispensed with the entire section on the $G M_{n}$. However this is not the case, as the results are used in the later proofs.

$\dagger$ In order to treat a function such as $w-\log z$, near a point at which $w-\log z=0$ we use the branch which vanishes at the point.

$\ddagger F^{1}$ and $F^{2}$ are equivalent at $P$ if $F^{1}=F^{2} \Omega$ at and near $P$, where $\Omega$ is analytic and not zero at $P$. 
From this definition it is evident that the topological properties of the R. M. S. are independent of any change in coordinates. In order to carry through our later proofs we make a change of coordinates if necessary, so that for no $\left(z^{0}\right)$ is $F\left(w, z^{0}\right)$ zero for all $w$ neighboring any value determining a point in $R$. That this can be done follows easily from a theorem of the authors dealing with a somewhat similar situation in the case of reals.*

Given a point $\left(w^{0}, z^{0}\right)$, with $F\left(w^{0}, z^{0}\right)=0$, we apply the Weierstrass Preparation Theorem $\dagger$, giving us, near $\left(w^{0}, z^{0}\right)$,

$$
F(w, z) \equiv \prod_{k}\left[F_{k}(w, z)\right] \Omega(w, z) .
$$

Here $\Omega$ is analytic and not zero at $\left(w^{0}, z^{0}\right)$, the product is finite, and $F_{k}$ is an irreducible algebroid polynomial, in general not singular, with vertex at $\left(z^{0}\right)$. Thus $F_{k}$ has the general form

$$
F_{k}(w, z)=w^{N}+\psi_{1}(z) w^{N-1}+\cdots+\psi_{N}(z),
$$

where the $\psi$ 's are analytic at $\left(z^{0}\right)$, and all the roots of $F_{k}$ coincide in the value $w^{0}$ when $(z)=\left(z^{0}\right)$.

From the properties of algebroid polynomials $\ddagger$ it follows that these $F_{k}$ 's can be taken as those mentioned in the definition of R. M. S. No two of them are equivalent, since in that case they would be identical and from the hypotheses on $F$ and $R$ it would follow that $F(w, z)$ would be reducible, contrary to hypothesis.

We observe that to each point of the locus $F=0$ correspond one or more (but a finite number of) points of the R. M. S. The points of the R. M. S. shall at times be considered in association with the corresponding points in $(w, z)$-space on the locus $F=0$, and at other times, as is ordinarily the case when $n=1$, in association with the corresponding points in (z)-space.

According to Theorem 6.II of $\mathrm{KB}$, if any closed sub-set of $R$ is given, a complex $K_{2 n+2}$ can be found containing the sub-set, such that the locus $F=0$ in $K_{2 n+2}$ is a sub-complex of even dimension, with analytic cells. In this case the dimension is $2 n$, and we denote the sub-complex by $K_{2 n}$. We denote by $K_{2 n-2}$ the complex of all cells of $K_{2 n}$ of dimensions less than $2 n-1$.

* On the covering of analytic loci by complexes, these Transactions, vol. 34 (1932), pp. 231-251; Theorem 5.I. We shall refer to this paper as KB. On p. 233 of this paper the words "In irreducible-C factorization" should be inserted at the beginning of the last sentence in Theorem 2.V, and also in Corollary 2.VI. In the last line on p. 233 the words "at the same points as" should be replaced by "identically if and only if the same is true for."

See also S. Lefschetz and J. H. C. Whitehead, Analytical complexes, these Transactions, vol. 35 (1933), pp. 510-517; \$4.

† Osgood II, chapter 2, \$2.

$\ddagger$ Osgood II, chapter $2, \S \S 5,7$. 
We shall now state and prove a simple set of rules for determining the R. M. S., and shall later use these rules in establishing c.rtain properties of the R. M. S.

LEMMA 4. The R. M.S. can be determined from $K_{2 n}$ as the locus $L_{2 n}$ which we now describe. We keep all of the $2 n$ - and $(2 n-1)$-cells of $K_{2 n}$. At each point, say $T$, of any cell $E_{p}, p<2 n-1$, we consider all the incident $(2 n-1)-$ and $2 n$ cells, and using them alone apply the test described in the concluding sentence of Lemma 3 to the neighborhood of $T$, finding that the incident $2 n$ - and $(2 n-1)$ cells are thus grouped into a finite number of sets, for each of which the condition of Lemma 3 is satisfied. For each of these sets we assign a point to $L_{2 n}$, corresponding to $T$. Then $L_{2 n}$ consists of $\left(K_{2 n}-K_{2 n-2}\right)$ and these new points, with neighborhood on $L_{2 n}$ determined as on $K_{2 n}$ except at points corresponding to points on $K_{2 n-2}$, where it is determined in an obvious manner by use of the incident $2 n$ and $(2 n-1)$-cells appearing in the tests mentioned above.

In applying this procedure at the boundary of $K_{2 n+2}$, we must consider $K_{2 n}$ enlarged by the addition of part of the locus $F=0$ outside of $K_{2 n+2}$.

Before proving the lemma we observe that we shall show later that $L_{2 n}$ is a complex and that, as we should expect from the above lemma, corresponding to each cell of $K_{2 n-2}$ is a finite positive number of cells of $L_{2 n}$; but corresponding to each cell of $K_{2 n}-K_{2 n-2}$ is just one cell of $L_{2 n}$.

We begin by observing that each point of $K_{2 n}-K_{2 n-2}$ yields just one point of the R. M. S.: the locus in $(z)$-space where values of $w$ coincide is defined by equating discriminants to zero, hence is at most $(2 n-2)$-dimensional. If any point of a $2 n$-cell or of a $(2 n-1)$-cell projected onto that locus, every point of the cell would project onto the locus, as all points of a cell have similar neighborhoods, and the cells project in one-to-one manner onto cells of the $(z)$-space. (The cells are obtained from cells in the (z)-space by two successive steps of the kind described on page 249 of $\mathrm{KB}$, where at each step we obtain a cell of the first class.) Since the locus in question in (z)-space is at most $(2 n-2)$-dimensional, we would then have a coritradiction to the invariance of dimensionality. ${ }^{*}$ Consequently, at each point of $K_{2 n}-K_{2 n-2}$, w is a single-valued, and hence analytic, function of the $z$ 's, and according to the definition of R. M. S. each such point therefore yields just one point of the R. M. S.

Now consider the points of the R. M. S. corresponding to a given point $P$ of $K_{2 n-2}$. For each of the irreducible functions $F^{i}$, vanishing at $P$, into which $F$ factors, we obtain a point on the R. M. S. Let $P^{0}$ be the projection of $P$ on $(z)$-space. Analytic continuation in a neighborhood of $P^{0}$, avoiding

\footnotetext{
* Brouwer. See Lefschetz I for references.
} 
points where the discriminant of $F$ vanishes, never leads from one function $F^{i}$ to a distinct function $F^{i}$, and furthermore such continuation can be made a test for distinguishing the functions $F^{i}$. In so testing, the paths can be made to avoid the projection, $K_{2 n-2}^{1}$, on $(z)$-space of $K_{2 n-2}$ without affecting the results, since any path avoiding points where the discriminant of $F$ vanishes can be deformed into a path of the kind wanted in such a way that none of the intermediate positions of the path pass through any point of the part of $K_{2 n-2}^{1}$ for which the discriminant vanishes. This is because $(z)$-space is $2 n$ dimensional. Consequently, for each point of the R. M. S. corresponding to $P$ the part of the R. M. S. corresponding to $\left(K_{2 n}-K_{2 n-2}\right)$ hangs together near the point in the way described in Lemma 3 , and must therefore be one of the sets designated in Lemma 4 . This proves that the process of Lemma 4 determines all of the points of the R. M. S. corresponding to $P$, and each on the boundary of the proper cells of $\left(K_{2 n}-K_{2 n-2}\right)$.

Since each such set of cells of $\left(K_{2 n}-K_{2 n-2}\right)$ must determine one of the functions $F^{i}$, it follows that no unwanted points are determined by the process of Lemma 4.

Consequently we have exactly the R. M. S. determined, and the proof of Lemma 4 is complete.

Lemma 5. The R. M. S. (locus $L_{2 n}$ ) is a complex.

We begin with the cells of $K_{2 n}-K_{2 n-2}$, which can be taken as part of a representation of $L_{2 n}$, as we have already seen. Now consider points of $L_{2 n}$ arising from points of $K_{2 n-2}$, in the light of Lemma 4. All points of a given cell of $K_{2 n-2}$ have similar neighborhoods on $K_{2 n}-K_{2 n-2}$, in fact, neighborhoods which are composed of parts of the same cells. From that fact and Lemma 4 it follows that corresponding to each cell of $K_{2 n-2}$ we have a finite number of cells of points of $L_{2 n}$, each incident with certain of the cells of higher dimension of $K_{2 n}-K_{2 n-2}$. Now $L_{2 n}$ is closed, as follows upon consideration of Lemma 4 , and of the fact that if a given cell of a complex is incident with certain cells of higher dimensions, then any cell on its boundary is incident with these cells of higher dimensions. Consequently $L_{2 n}$ is a complex, as we wished to prove.

Theorem 1. The Riemann multiple-space $\left(L_{2 n}\right)$ is a set of generalized manifolds $\left(\bmod\right.$ boundary of $\left.K_{2 n+2}\right)$.

By this we mean that it is a complex consisting of a number of parts each

* Osgood II, chapter $2, \S \S 10,11$. We do not find there a general treatment of R.M.S., as the points for which the discriminant vanishes are not treated. 
of which satisfies the definition of generalized manifold except at the boundary of $K_{2 n+2}$.

According to Lemmas 5, 4 and 3, $L_{2 n}$ satisfies the condition for a set of generalized manifolds, except at the boundary of $K_{2 n+2}$. Consequently Theorem 1 is valid.

4. Non-spherical points. Any point of $L_{2 n}$ which does not have a neighborhood on the R. M. S. homeomorphic to a $2 n$-cell shall be called a non-spherical point. We shall prove that the non-spherical points form a sub-complex of dimension not greater than $2 n-4$.

Theorem 2. The R. M. S. $L_{2 n}$ can be formed from $K_{2 n}$ (locus $F=0$ ), by the process described by Veblen, ${ }^{*}$ used in his proof that every n-circuit is a singular generalized n-manifold.

With the $2 n$ - and $(2 n-1)$-cells we get the correct result, since each $(2 n-1)$-cell not on the boundary of $K_{2 n+2}$ is incident with just two $2 n$-cells. Now we use induction, supposing that we have got the correct result with all cells down to those of dimension $p+1$, and next consider those of dimension $p$. Under each of the two methods, that given by Veblen and that given in Lemma 4 (under the test of Lemma 3), we replace a given $p$-cell $E_{p}$ of $K_{2 n-2}$ by a finite number of $p$-cells, each incident with certain groups of cells of higher dimensions. In the first case, we have one $p$-cell for each group of incident cells of higher dimensions which remain connected near $E_{p}$ when $E_{p}$ is removed, and in the second case we have a similar test, but consider only the incident cells of dimensions $2 n$ and $2 n-1$. But from the corollary to Lemma 3 we see that, since we know that we have a generalized manifold insofar as cells of dimensions greater than $p$ are tested, we obtain the same result by each of the two methods. Consequently Theorem 2 is valid.

Theorem 3. The non-spherical points of the R. M.S. $\left(L_{2 n}\right)$ form a subcomplex of dimension at most $2 n-4$.

As the set of spherical points is evidently an open set on $L_{2 n}$, the set of non-spherical points must be closed. As it must consist of a certain number of cells, it is therefore a complex. It remains to prove that this complex is of dimension at most $2 n-4$.

It is shown in $\mathrm{KB} \dagger$ that near any point $P$ on the locus $F=0$, above a point where the discriminant is zero, but where the discriminant of the discriminant is not zero (upon second application of the Weierstrass Preparation Theorem), the locus of similarly described points near $P$ is obtained by equating

* Loc. cit.

$\dagger \S 4$, pp. 236-242. 
$w$ and $z_{n}$ each to an analytic function of $\left(z_{1}, \cdots, z_{n-1}\right)$. We denote by $J_{2 n-2}$ a $(2 n-2)$-cell neighborhood of $P$ consisting of such similar points. Let $J_{2 n-2}^{1}$ denote the projection on ( $z$-space of $J_{2 n-2}$, with equation $z_{n}=\psi\left(z_{1}, \cdots, z_{n-1}\right)$. We now cover a neighborhood of the points of $J_{2 n-2}^{1}$ in $(z)$-space by a set of analytic cells of dimensions $2 n-1$ and $2 n$, as follows. Let $E_{2 n-2}$ be a flat cell, part of the locus $y_{n}=0$ in the $2 n$-space of the complex variables $\left(y_{1}, \cdots, y_{n}\right)$. Cover a neighborhood of $E_{2 n-2}$ in (y)-space by $E_{2 n-2}$ and a set of flat (2n-1)cells and $2 n$-cells, each incident with $E_{2 n-2}$, alternating in order, arranged in cyclic order. Next make the transformation (with non-vanishing Jacobian) $y_{i}=z_{i}, i=1, \cdots, n-1$, and $y_{n}=z_{n}-\psi\left(z_{1}, \cdots, z_{n-1}\right)$. This transformation gives us the set of cells covering a neighborhood of $J_{2 n-2}^{1}$, that we wanted. Above any of the $2 n$ - or $(2 n-1)$-cells of this neighborhood, near $J_{2 n-2}$, w equals a finite number of distinct-valued analytic functions of $(z)=\left(z_{1}, \cdots\right.$, $\left.z_{n}\right)$, since these cells contain no points for which the discriminant vanishes. Corresponding to a circuit of the $2 n$ - and $(2 n-1)$-cells incident with $E_{2 n-2}$ in (y)-space we will now have a circuit around $J_{2 n-2}$ (we can consider a curve going around it), and if we go around enough times (a finite number) we must come back to the original value of $w$, hence back to the original point of the R. M. S. at which we started the curve. Hence the point $P$ has a neighborhood on $L_{2 n}$ consisting of $J_{2 n-2}$ and a set of incident $2 n$ - and $(2 n-1)$-cells (not cells of $L_{2 n}$ ) arranged in cyclic order, and alternating. Of course, $P$ might be on a $(2 n-3)$-cell of $L_{2 n}$, or even on one of lower dimension, but that does not affect our work. Consequently $P$ is a spherical point.

Therefore the non-spherical points of $L_{2 n}$ must project onto points of $(z)$ space for which the discriminant of the discriminant is zero. The locus of such points is at most $(2 n-4)$-dimensional, and hence the locus of non-spherical points cannot contain any cell of dimension higher than $2 n-4$. For such a cell would project onto $(z)$-space in a cell of the same dimension $\geqq 2 n-3$, which would contradict the result just obtained. Thus the proof is complete.

Columbia University, NEW YORK, N. Y. 